International Journal of Pure and Applied Mathematics

Volume 105 No. 2 2015, 257-268

ISSN: 1311-8080 (printed version); ISSN: 1314-3395 (on-line version)

url: http://www.ijpam.eu

doi: http://dx.doi.org/10.12732/ijpam.v105i2.11

ijpam.eu

\title{
BOUNDS FOR A TOADER-TYPE MEAN BY ARITHMETIC AND CONTRAHARMONIC MEANS
}

\author{
Nali $\mathrm{Li}^{1}$, Tiehong Zhao ${ }^{2}$, Yile Zhao ${ }^{3}$ \\ ${ }^{1,2}$ Department of Mathematics \\ Hangzhou Normal University \\ Hangzhou, 311121, P.R. CHINA
}

Abstract: In this paper, we present the best possible parameters $\alpha_{i}$ and $\beta_{i}$ with $i=1,2,3$, 4 such that the double inequalities

$$
\begin{aligned}
& \alpha_{1} A(a, b)+\left(1-\alpha_{1}\right) C(a, b)<T[A(a, b), C(a, b)]<\beta_{1} A(a, b)+\left(1-\beta_{1}\right) C(a, b), \\
& A^{\alpha_{2}}(a, b) C^{1-\alpha_{2}}(a, b)<T[A(a, b), C(a, b)]<A^{\beta_{2}}(a, b) C^{1-\beta_{2}}(a, b), \\
& \frac{\alpha_{3}}{A(a, b)}+\frac{1-\alpha_{3}}{C(a, b)}<\frac{1}{T[A(a, b), C(a, b)]}<\frac{\beta_{3}}{A(a, b)}+\frac{1-\beta_{3}}{C(a, b)}, \\
& C\left[\alpha_{4} a+\left(1-\alpha_{4}\right) b, \alpha_{4} b+\left(1-\alpha_{4}\right) a\right]<T[A(a, b), C(a, b)] \\
& <C\left[\beta_{4} a+\left(1-\beta_{4}\right) b, \beta_{4} b+\left(1-\beta_{4}\right) a\right]
\end{aligned}
$$

hold for all $a, b>0$ with $a \neq b$, as consequences, we provide several new bounds for the complete elliptic integral $\mathcal{E}(r)=\int_{0}^{\pi / 2}\left(1-r^{2} \sin ^{2} \theta\right)^{1 / 2} d \theta(r \in$ $(0, \sqrt{3} / 2)$ of the second kind, where $T(a, b)=\frac{2}{\pi} \int_{0}^{\pi / 2} \sqrt{a^{2} \cos ^{2} \theta+b^{2} \sin ^{2} \theta} d \theta$, $A(a, b)=(a+b) / 2$ and $C(a, b)=\left(a^{2}+b^{2}\right) /(a+b)$ are the Toader, arithmetic and contraharmonic means of $a$ and $b$, respectively.

AMS Subject Classification: 26E60

Key Words: arithmetic mean, Toader mean, contraharmonic mean

Received: September 20, 2015

(c) 2015 Academic Publications, Ltd. url: www.acadpubl.eu

$\S_{\text {Correspondence author }}$ 


\section{Introduction}

For $a, b>0$ with $a \neq b$, let $p \in[0,1]$ and $q \in \mathbb{R}$, then the $p$-th generalized Seiffert mean $S_{p}(a, b), q$-th Gini mean $G_{q}(a, b), q$-th power mean $M_{q}(a, b), q$-th Lehmer mean $L_{q}(a, b)$, harmonic mean $H(a, b)$, geometric mean $G(a, b)$, quadratic mean $Q(a, b)$, centroidal mean $\bar{C}(a, b)$, arithmetic mean $A(a, b)$ and contraharmonic mean $C(a, b)$ are respectively defined by

$$
\begin{aligned}
& S_{p}(a, b)=\left\{\begin{array}{lr}
\frac{p(a-b)}{\arctan \left[\frac{2 p(a-b)}{a+b}\right]}, & 0<p \leq 1, \\
\frac{a+b}{2}, & p=0,
\end{array}\right. \\
& G_{q}(a, b)= \begin{cases}\left(\frac{a^{q-1}+b^{q-1}}{a+b}\right)^{1 /(q-2)}, & q \neq 2, \\
\left(a^{a} b^{b}\right)^{1 /(a+b)}, & q=2,\end{cases} \\
& M_{q}(a, b)= \begin{cases}\left(\frac{a^{q}+b^{q}}{2}\right)^{1 / q}, & q \neq 0, \\
\sqrt{a b}, & q=0,\end{cases} \\
& L_{q}(a, b)=\frac{a^{q+1}+b^{q+1}}{a^{q}+b^{q}}, \quad H(a, b)=\frac{2 a b}{a+b}, \quad G(a, b)=\sqrt{a b}, \\
& Q(a, b)=\sqrt{\frac{a^{2}+b^{2}}{2}}, \quad \bar{C}(a, b)=\frac{2\left(a^{2}+a b+b^{2}\right)}{3(a+b)} \\
& A(a, b)=\frac{a+b}{2}, \quad C(a, b)=\frac{a^{2}+b^{2}}{a+b} .
\end{aligned}
$$

It is well known that $S_{p}(a, b), G_{q}(a, b), M_{q}(a, b)$ and $L_{q}(a, b)$ are continuous and strictly increasing with respect to $p \in[0,1]$ and $q \in \mathbb{R}$ for fixed $a, b>0$ with $a \neq b$, and the inequality chain

$$
\begin{gathered}
H(a, b)=M_{-1}(a, b)=L_{-1}(a, b)<G(a, b)=M_{0}(a, b)=L_{-1 / 2}(a, b) \\
<A(a, b)=M_{1}(a, b)=L_{0}(a, b)<T(a, b)<\bar{C}(a, b) \\
<Q(a, b)=M_{2}(a, b)<C(a, b)=L_{1}(a, b)
\end{gathered}
$$

hold for all $a, b>0$ with $a \neq b$.

In [1], Toader introduced the Toader mean $T(a, b)$ of two positive numbers $a$ and $b$ as follows:

$$
T(a, b)=\frac{2}{\pi} \int_{0}^{\pi / 2} \sqrt{a^{2} \cos ^{2} \theta+b^{2} \sin ^{2} \theta} d \theta
$$




$$
= \begin{cases}\frac{2 a}{\pi} \mathcal{E}\left(\sqrt{1-\left(\frac{b}{a}\right)^{2}}\right), & a>b, \\ \frac{2 b}{\pi} \mathcal{E}\left(\sqrt{1-\left(\frac{a}{b}\right)^{2}}\right), & a<b, \\ a, & a=b,\end{cases}
$$

where $\mathcal{E}(r)=\int_{0}^{\pi / 2}\left(1-r^{2} \sin ^{2} \theta\right)^{1 / 2} d \theta(r \in[0,1])$ is the complete elliptic integral of the second kind.

Recently, the Toader mean $T(a, b)$ has been the subject of intensive research. Vuorinen [2] conjectured that the inequality

$$
M_{3 / 2}(a, b)<T(a, b)
$$

holds for all $a, b>0$ with $a \neq b$. This conjecture was proved by Qiu and Shen [3], and Barnard et al. [4], respectively.

Alzer and Qiu [5] presented a best possible upper power mean bound for the Toader mean as follows:

$$
T(a, b)<M_{\log 2 /(\log \pi-\log 2)}(a, b)
$$

for all $a, b>0$ with $a \neq b$.

Neuman [6], and Kazi and Neuman [7] proved that the inequalities

$$
\begin{gathered}
\frac{(a+b) \sqrt{a b}-a b}{A G M(a, b)}<T(a, b)<\frac{4(a+b) \sqrt{a b}+(a-b)^{2}}{8 A G M(a, b)} \\
T(a, b)<\frac{1}{4}\left(\sqrt{(2+\sqrt{2}) a^{2}+(2-\sqrt{2}) b^{2}}+\sqrt{(2+\sqrt{2}) b^{2}+(2-\sqrt{2}) a^{2}}\right)
\end{gathered}
$$

hold for all $a, b>0$ with $a \neq b$, where $\operatorname{AGM}(a, b)$ is the arithmetic-geometric mean of $a$ and $b$.

In [8-10], the best possible parameters $\lambda_{1}, \mu_{1} \in[0,1]$ and $\lambda_{2}, \mu_{2}, \lambda_{3}, \mu_{3} \in \mathbb{R}$ such that the double inequalities $S_{\lambda_{1}}(a, b)<T(a, b)<S_{\mu_{1}}(a, b), G_{\lambda_{2}}(a, b)<$ $T(a, b)<G_{\mu_{2}}(a, b)$ and $L_{\lambda_{3}}(a, b)<T(a, b)<L_{\mu_{3}}(a, b)$ hold for all $a, b>0$ with $a \neq b$ were presented.

In [11-15], the authors proved that the double inequalities

$$
\begin{aligned}
\alpha_{1} Q(a, b)+\left(1-\alpha_{1}\right) A(a, b) & <T(a, b)<\beta_{1} Q(a, b)+\left(1-\beta_{1}\right) A(a, b), \\
Q^{\alpha_{2}}(a, b) A^{\left(1-\alpha_{2}\right)}(a, b) & <T(a, b)<Q^{\beta_{2}}(a, b) A^{\left(1-\beta_{2}\right)}(a, b), \\
\alpha_{3} C(a, b)+\left(1-\alpha_{3}\right) A(a, b)<T(a, b) & <\beta_{3} C(a, b)+\left(1-\beta_{3}\right) A(a, b),
\end{aligned}
$$




$$
\begin{aligned}
& \frac{\alpha_{4}}{A(a, b)}+\frac{1-\alpha_{4}}{C(a, b)}<\frac{1}{T(a, b)}<\frac{\beta_{4}}{A(a, b)}+\frac{1-\beta_{4}}{C(a, b)}, \\
& \alpha_{5} C(a, b)+\left(1-\alpha_{5}\right) H(a, b)<T(a, b)<\beta_{5} C(a, b)+\left(1-\beta_{5}\right) H(a, b), \\
& \alpha_{6}[C(a, b)-H(a, b)]+A(a, b)<T(a, b)<\beta_{6}[C(a, b)-H(a, b)]+A(a, b), \\
& \alpha_{7} \bar{C}(a, b)+\left(1-\alpha_{7}\right) A(a, b)<T(a, b)<\beta_{7} \bar{C}(a, b)+\left(1-\beta_{7}\right) A(a, b), \\
& \frac{\alpha_{8}}{A(a, b)}+\frac{1-\alpha_{8}}{\bar{C}(a, b)}<\frac{1}{T(a, b)}<\frac{\beta_{8}}{A(a, b)}+\frac{1-\beta_{8}}{\bar{C}(a, b)}, \\
& \alpha_{9} Q(a, b)+\left(1-\alpha_{9}\right) H(a, b)<T(a, b)<\beta_{9} Q(a, b)+\left(1-\beta_{9}\right) H(a, b), \\
& \frac{\alpha_{10}}{H(a, b)}+\frac{1-\alpha_{10}}{Q(a, b)}<\frac{1}{T(a, b)}<\frac{\beta_{10}}{H(a, b)}+\frac{1-\beta_{10}}{Q(a, b)}
\end{aligned}
$$

hold for all $a, b>0$ with $a \neq b$ if and only if $\alpha_{1} \leq 1 / 2, \beta_{1} \geq(4-\pi) /[(\sqrt{2}-1) \pi]$, $\alpha_{2} \leq 1 / 2, \beta_{2} \geq 4-2 \log \pi / \log 2, \alpha_{3} \leq 1 / 4, \beta_{3} \geq 4 / \pi-1, \alpha_{4} \leq \pi / 2-1, \beta_{4} \geq 3 / 4$, $\alpha_{5} \leq 5 / 8, \beta_{5} \geq 2 / \pi, \alpha_{6} \leq 1 / 8, \beta_{6} \geq 2 / \pi-1 / 2, \alpha_{7} \leq 3 / 4, \beta_{7} \geq 12 / \pi-3$, $\alpha_{8} \leq \pi-3, \beta_{8} \geq 1 / 4, \alpha_{9} \leq 5 / 6, \beta_{9} \geq 2 \sqrt{2} / \pi, \alpha_{10} \leq 0$ and $\beta_{10} \geq 1 / 6$.

Moreover, Chu et al. [16] and Hua and Qi [17] proved that the double inequalities

$$
\begin{aligned}
& C[\lambda a+(1-\lambda) b, \lambda b+(1-\lambda) a]<T(a, b)<C[\mu a+(1-\mu) b, \mu b+(1-\mu) a], \\
& \bar{C}[\alpha a+(1-\alpha) b, \alpha b+(1-\alpha) a]<T(a, b)<\bar{C}[\beta a+(1-\beta) b, \beta b+(1-\beta) a]
\end{aligned}
$$

hold for all $a, b>0$ with $a \neq b$ if and only if $\lambda \leq 3 / 4, \mu \geq 1 / 2+\sqrt{\pi(4-\pi)} /(2 \pi)$, $\alpha \leq 1 / 2+\sqrt{3} / 4$ and $\beta \geq 1 / 2+\sqrt{12 / \pi-3} / 2$.

The main purpose of this paper is to present the best possible parameters $\alpha_{1}, \alpha_{2}, \alpha_{3}, \alpha_{4}$ and $\beta_{1}, \beta_{2}, \beta_{3}, \beta_{4}$ such that the double inequalities

$$
\begin{aligned}
& \alpha_{1} A(a, b)+\left(1-\alpha_{1}\right) C(a, b)<T[A(a, b), C(a, b)]<\beta_{1} A(a, b)+\left(1-\beta_{1}\right) C(a, b), \\
& A^{\alpha_{2}}(a, b) C^{1-\alpha_{2}}(a, b)<T[A(a, b), C(a, b)]<A^{\beta_{2}}(a, b) C^{1-\beta_{2}}(a, b), \\
& \frac{\alpha_{3}}{A(a, b)}+\frac{1-\alpha_{3}}{C(a, b)}<\frac{1}{T[A(a, b), C(a, b)]}<\frac{\beta_{3}}{A(a, b)}+\frac{1-\beta_{3}}{C(a, b)}, \\
& C\left[\alpha_{4} a+\left(1-\alpha_{4}\right) b, \alpha_{4} b+\left(1-\alpha_{4}\right) a\right]<T[A(a, b), C(a, b)] \\
& <C\left[\beta_{4} a+\left(1-\beta_{4}\right) b, \beta_{4} b+\left(1-\beta_{4}\right) a\right]
\end{aligned}
$$

hold for all $a, b>0$ with $a \neq b$. 


\section{Basic Knowledge and Lemmas}

In order to prove our main results we need several formulas and lemmas, which we present in this section.

For $r \in(0,1)$ and $r^{\prime}=\sqrt{1-r^{2}}$, the well-known complete elliptic integrals of the first and second kinds are defined by

$$
\left\{\begin{array}{l}
\mathcal{K}=\mathcal{K}(r)=\int_{0}^{\pi / 2}\left(1-r^{2} \sin ^{2} \theta\right)^{-1 / 2} d \theta \\
\mathcal{K}^{\prime}=\mathcal{K}^{\prime}(r)=\mathcal{K}\left(r^{\prime}\right) \\
\mathcal{K}(0)=\pi / 2, \quad \mathcal{K}(1)=+\infty
\end{array}\right.
$$

and

$$
\left\{\begin{array}{l}
\mathcal{E}=\mathcal{E}(r)=\int_{0}^{\pi / 2}\left(1-r^{2} \sin ^{2} \theta\right)^{1 / 2} d \theta \\
\mathcal{E}^{\prime}=\mathcal{E}^{\prime}(r)=\mathcal{E}\left(r^{\prime}\right) \\
\mathcal{E}(0)=\pi / 2, \quad \mathcal{E}(1)=1,
\end{array}\right.
$$

respectively, and the following formulas were presented in [18, Appendix E, pp. 474-475]:

$$
\begin{gathered}
\frac{d \mathcal{K}}{d r}=\frac{\mathcal{E}-r^{\prime 2} \mathcal{K}}{r r^{\prime 2}}, \quad \frac{d \mathcal{E}}{d r}=\frac{\mathcal{E}-\mathcal{K}}{r}, \\
\frac{d\left(\mathcal{E}-r^{\prime 2} \mathcal{K}\right)}{d r}=r \mathcal{K}, \quad \frac{d(\mathcal{K}-\mathcal{E})}{d r}=\frac{r \mathcal{E}}{r^{\prime 2}} \\
\mathcal{E}\left(\frac{2 \sqrt{r}}{1+r}\right)=\frac{2 \mathcal{E}-r^{\prime 2} \mathcal{K}}{1+r} .
\end{gathered}
$$

In what follows, two special values $\mathcal{E}(1 / 3)$ and $\mathcal{K}(1 / 3)$ will be used. By numerical computations, these are given by

$$
\mathcal{E}(1 / 3)=1.52621 \cdots, \quad \mathcal{K}(1 / 3)=1.61739 \cdots .
$$

Lemma 2.1. (See [18, Theorem 1.25]). For $-\infty<a<b<\infty$, let $f, g:[a, b] \rightarrow \mathbb{R}$ be continuous on $[a, b]$, and be differentiable on $(a, b)$, let $g^{\prime}(x) \neq 0$ on $(a, b)$. If $f^{\prime}(x) / g^{\prime}(x)$ is increasing (decreasing) on $(a, b)$, then so are

$$
\frac{f(x)-f(a)}{g(x)-g(a)} \quad \text { and } \quad \frac{f(x)-f(b)}{g(x)-g(b)} .
$$

If $f^{\prime}(x) / g^{\prime}(x)$ is strictly monotone, then the monotonicity in the conclusion is also strict.

Lemma 2.2. Let $r \in(0,1)$, then 
itemindent $=-2 \mathrm{em}$ the function $r \rightarrow\left(\mathcal{E}-r^{\prime 2} \mathcal{K}\right) / r^{2}$ is strictly increasing from $(0,1)$ onto $(\pi / 4,1)$;

iitemiindent $=-2 \mathrm{em}$ the function $r \rightarrow 2 \mathcal{E}-r^{\prime 2} \mathcal{K}$ is increasing and log-convex from $(0,1)$ onto $(\pi / 2,2)$.

Proof. Parts (1) and (2) follow from [18, Theorem 3.21(1) and Exercise $3.43(13)]$.

Lemma 2.3. Let $r \in(0,1 / 3), \lambda_{1}=2\left[1-\frac{3}{\pi} \mathcal{E}(1 / 3)+\frac{4}{3 \pi} \mathcal{K}(1 / 3)\right]=0.458 \cdots$ and

$$
f(r)=\frac{1+r-\frac{2}{\pi}\left(2 \mathcal{E}-r^{\prime 2} \mathcal{K}\right)}{2 r}
$$

then $f(r)$ is strictly decreasing from $(0,1 / 3)$ onto $\left(\lambda_{1}, 1 / 2\right)$.

Proof. Let $f_{1}(r)=1+r-2\left(2 \mathcal{E}-r^{\prime} \mathcal{K}\right) / \pi$ and $f_{2}(r)=2 r$. Then $f_{1}(0)=$ $f_{2}(0)=0$ and $f(r)=f_{1}(r) / f_{2}(r)$. Differentiation of $f_{1}(r)$ and $f_{2}(r)$ yields

$$
\frac{f_{1}^{\prime}(r)}{f_{2}^{\prime}(r)}=\frac{1}{2}-\frac{r}{\pi} \cdot \frac{\mathcal{E}-r^{\prime} \mathcal{K}}{r^{2}}
$$

It follows from Lemma $2.2(1)$ that $f_{1}^{\prime}(r) / f_{2}^{\prime}(r)$ is strictly decreasing in $(0,1)$.

Therefore, Lemma 2.3 follows from Lemma 2.1 and the limiting values $f\left(0^{+}\right)=\frac{1}{2}$ and $f\left(\frac{1}{3}^{-}\right)=\lambda_{1}$.

Lemma 2.4. Let $r \in(0,1 / 3), \lambda_{2}=\log [3 \pi /(9 \mathcal{E}(1 / 3)-4 \mathcal{K}(1 / 3))] / \log 2=$ $0.375 \cdots$ and

$$
g(r)=\frac{\log \left(4 \mathcal{E}-2 r^{\prime} \mathcal{K}\right)-\log [\pi(1+r)]}{\log (1-r)-\log (1+r)},
$$

then $g(r)$ is strictly decreasing from $(0,1 / 3)$ onto $\left(\lambda_{2}, 1 / 2\right)$.

Proof. Let $g_{1}(r)=\log \left(4 \mathcal{E}-2 r^{\prime} 2 \mathcal{K}\right)-\log [\pi(1+r)]$ and $g_{2}(r)=\log (1-r)-$ $\log (1+r)$ Then $g_{1}(0)=g_{2}(0)=0, g(r)=g_{1}(r) / g_{2}(r)$ and

$$
\frac{g_{1}^{\prime}(r)}{g_{2}(r)}=g_{3}(r) g_{4}(r) \text {. }
$$

where

$$
g_{3}(r)=\frac{(1-r)^{2}[(1+r) \mathcal{K}-\mathcal{E}]}{r}, \quad g_{4}(r)=\frac{1}{2\left(2 \mathcal{E}-r^{\prime 2} \mathcal{K}\right)} .
$$

A simple calculation yields

$$
g_{3}^{\prime}(r)=-\frac{(1-r)\left[(1+2 r)(\mathcal{K}-\mathcal{E})+r^{2} \mathcal{K}\right]}{r^{2}}<0
$$


for $r \in(0,1)$.

It follows from (2.5) and Lemma 2.2(2) that $g_{3}(r)$ is strictly decreasing from $(0,1)$ onto $(0, \pi / 2)$ and $g_{4}(r)$ is strictly decreasing from $(0,1)$ onto $(1 / 4,1 / \pi)$. This conjunction with $(2.4)$ implies that $g_{1}^{\prime}(r) / g_{2}^{\prime}(r)$ is strictly decreasing in $(0,1)$.

Therefore, Lemma 2.4 follows from Lemma 2.1 and the limiting values $g\left(0^{+}\right)=\frac{1}{2}$ and $g\left(\frac{1}{3}^{-}\right)=\lambda_{2}$.

Lemma 2.5. Let $r \in(0,1 / 3), \lambda_{3}=[3 \pi-9 \mathcal{E}(1 / 3)+4 \mathcal{K}(1 / 3)] /[9 \mathcal{E}(1 / 3)-$ $4 \mathcal{K}(1 / 3)]=0.297 \cdots$ and

$$
h(r)=\frac{\pi r^{\prime 2}-2(1-r)\left(2 \mathcal{E}-r^{\prime 2} \mathcal{K}\right)}{4 r\left(2 \mathcal{E}-r^{\prime 2} \mathcal{K}\right)}
$$

then $h(r)$ is strictly decreasing from $(0,1 / 3)$ onto $\left(\lambda_{3}, 1 / 2\right)$.

Proof. Let $\widehat{h}(r)=\left[\pi r^{\prime 2}-2(1-r)\left(2 \mathcal{E}-r^{\prime 2} \mathcal{K}\right)\right] / r$, then $h(r)=\widehat{h}(r) /[4(2 \mathcal{E}-$ $\left.\left.r^{\prime 2} \mathcal{K}\right)\right]$. Following from Lemma 2.2(2), it suffices to prove that $\widehat{h}(r)$ is strictly decreasing in $(0,1 / 3)$.

Differentiation of $\widehat{h}(r)$ leads to

$$
\begin{aligned}
\widehat{h}^{\prime}(r) & =-\frac{\widehat{h}_{1}(r)}{r^{2}} \\
\widehat{h}_{1}(r) & =\pi\left(1+r^{2}\right)+2 r r^{2} \mathcal{K}-2(1+r) \mathcal{E}, \\
\widehat{h}_{1}(0) & =0 \\
\widehat{h}_{1}^{\prime}(r) & =\frac{2}{r}\left[r^{2}(\pi-2 r \mathcal{K})+(1+r)(\mathcal{K}-\mathcal{E})\right] .
\end{aligned}
$$

It is well-known that the function $\pi-2 r \mathcal{K}$ is strictly decreasing in $(0,1)$ and then $\pi-2 r \mathcal{K}>\pi-\frac{2}{3} \mathcal{K}(1 / 3)=2.063 \cdots>0$ for $0<r<1 / 3$. It is easy to see that $\widehat{h}_{1}^{\prime}(r)>0$ for $0<r<1 / 3$ following from (2.9). This conjunction with (2.6)-(2.8) implies that $\widehat{h}(r)$ is strictly decreasing in $(0,1 / 3)$.

Therefore, Lemma 2.5 follows from the limiting values $h\left(0^{+}\right)=\frac{1}{2}$ and $h\left(\frac{1}{3}^{-}\right)=\lambda_{3}$.

Lemma 2.6. Let $r \in(0,1 / 3), \lambda_{4}=[18 \mathcal{E}(1 / 3)-8 \mathcal{K}(1 / 3)] / 3 \pi-1=$ $0.5419 \cdots$ and

$$
\varphi(r)=\frac{\frac{2}{\pi}\left(2 \mathcal{E}-r^{\prime 2} \mathcal{K}\right)+r-1}{2 r},
$$

then $\varphi(r)$ is strictly increasing from $(0,1 / 3)$ onto $\left(1 / 2, \lambda_{4}\right)$. 
Proof. The proof completes easily from the following facts

$$
\varphi^{\prime}(r)=\frac{\frac{\pi}{2}-\mathcal{E}}{\pi r^{2}}>0
$$

for $r \in(0,1 / 3)$ and the limiting values $\varphi\left(0^{+}\right)=1 / 2$ and $\varphi\left(\frac{1}{3}^{-}\right)=\lambda_{4}$.

\section{Main Results}

Theorem 3.1. The double inequality

$$
\alpha_{1} A(a, b)+\left(1-\alpha_{1}\right) C(a, b)<T[A(a, b), C(a, b)]<\beta_{1} A(a, b)+\left(1-\beta_{1}\right) C(a, b)
$$

holds for all $a, b>0$ with $a \neq b$ if and only if $\alpha_{1} \geq 1 / 2$ and $\beta_{1} \leq \lambda_{1}=$ $2\left[1-\frac{3}{\pi} \mathcal{E}(1 / 3)+\frac{4}{3 \pi} \mathcal{K}(1 / 3)\right]=0.458 \cdots$.

Proof. It is well-known that $A(a, b), T(a, b)$ and $C(a, b)$ are symmetric and homogeneous of degree one. Without loss of generality, we assume that $a>b$. Let $r=(a-b)^{2} /\left(3 a^{2}+2 a b+3 b^{2}\right) \in(0,1 / 3)$. From (1.1), (1.2) and (2.2), we see that

$$
\begin{aligned}
A(a, b) & =C(a, b) \frac{1-r}{1+r} \\
T[A(a, b), C(a, b)] & =\frac{2}{\pi} C(a, b) \mathcal{E}\left(\frac{2 \sqrt{r}}{1+r}\right)=\frac{2}{\pi} C(a, b) \frac{2 \mathcal{E}-r^{\prime 2} \mathcal{K}}{1+r}
\end{aligned}
$$

and

$$
\frac{C(a, b)-T[A(a, b), C(a, b)]}{C(a, b)-A(a, b)}=\frac{1-\frac{2}{\pi} \frac{2 \mathrm{E}-r^{\prime 2} \mathrm{~K}}{1+r}}{1-\frac{1-r}{1+r}}=f(r),
$$

where $f(r)$ is defined as in Lemma 2.3.

Therefore, Theorem 3.1 follows easily from Lemma 2.3 and (3.3).

Theorem 3.2. The double inequality

$$
A^{\alpha_{2}}(a, b) C^{1-\alpha_{2}}(a, b)<T[A(a, b), C(a, b)]<A^{\beta_{2}}(a, b) C^{1-\beta_{2}}(a, b)
$$

holds for all $a, b>0$ with $a \neq b$ if and only if $\alpha_{2} \geq 1 / 2$ and $\beta_{2} \leq \lambda_{2}=$ $\log [3 \pi /(9 \mathcal{E}(1 / 3)-4 \mathcal{K}(1 / 3))] / \log 2=0.375 \cdots$. 
Proof. Without loss of generality, we assume that $a>b$. Let $r=(a-$ $b)^{2} /\left(3 a^{2}+2 a b+3 b^{2}\right) \in(0,1 / 3)$. Then (3.1) and (3.2) lead to

$$
\frac{\log C(a, b)-\log T[A(a, b), C(a, b)]}{\log C(a, b)-\log A(a, b)}=g(r),
$$

where $g(r)$ is defined as in Lemma 2.4.

Therefore, Theorem 3.2 follows easily from Lemma 2.4 and (3.4).

Theorem 3.3. The double inequality

$$
\frac{\alpha_{3}}{A(a, b)}+\frac{1-\alpha_{3}}{C(a, b)}<\frac{1}{T[A(a, b), C(a, b)]}<\frac{\beta_{3}}{A(a, b)}+\frac{1-\beta_{3}}{C(a, b)}
$$

holds for all $a, b>0$ with $a \neq b$ if and only if $\alpha_{3} \leq \lambda_{3}=[3 \pi-9 \mathcal{E}(1 / 3)+$ $4 \mathcal{K}(1 / 3)] /[9 \mathcal{E}(1 / 3)-4 \mathcal{K}(1 / 3)]=0.297 \cdots$ and $\beta_{3} \geq 1 / 2$.

Proof. Without loss of generality, we assume that $a>b$. Let $r=(a-$ $b)^{2} /\left(3 a^{2}+2 a b+3 b^{2}\right) \in(0,1 / 3)$. Then (3.1) and (3.2) lead to

$$
\frac{\frac{1}{T[A(a, b), C(a, b)]}-\frac{1}{C(a, b)}}{\frac{1}{A(a, b)}-\frac{1}{C(a, b)}}=h(r),
$$

where $h(r)$ is defined as in Lemma 2.5 .

Therefore, Theorem 3.3 follows easily from Lemma 2.5 and (3.5).

Theorem 3.4. The double inequality

$$
\begin{aligned}
C\left[\alpha_{4} a+\left(1-\alpha_{4}\right) b, \alpha_{4} b+\left(1-\alpha_{4}\right) a\right] & <T[A(a, b), C(a, b)] \\
& <C\left[\beta_{4} a+\left(1-\beta_{4}\right) b, \beta_{4} b+\left(1-\beta_{4}\right) a\right]
\end{aligned}
$$

holds for all $a, b>0$ with $a \neq b$ if and only if $\alpha_{4} \leq(2+\sqrt{2}) / 4$ and $\beta_{4} \geq$ $\left(1+\sqrt{\lambda_{4}}\right) / 2=0.868 \cdots$.

Proof. For positive numbers $a, b>0$ with $a \neq b$, let $J(x)=C[x a+(1-$ $x) b, x b+(1-x) a]$ be on $[1 / 2,1]$, then it is easy to verify that $J(x)$ is continuous and strictly increasing on $[1 / 2,1]$. Since $C(a, b)$ and $T(a, b)$ are symmetric and homogenous of degree one, we assume that $a>b$. Let $r=(a-b)^{2} /\left(3 a^{2}+2 a b+\right.$ $\left.3 b^{2}\right) \in(0,1 / 3)$ and $p \in(1 / 2,1)$. Then (3.1) and (3.2) lead to

$$
T[A(a, b), C(a, b)]-C[p a+(1-p) b, p b+(1-p) a]
$$




$$
\begin{aligned}
& =\frac{2}{\pi} C(a, b) \frac{2 \mathcal{E}-r^{\prime 2} \mathcal{K}}{1+r}-C(a, b) \frac{1-r+2(2 p-1)^{2} r}{1+r} \\
& =\frac{2 r C(a, b)}{1+r}\left[\varphi(r)-(2 p-1)^{2}\right],
\end{aligned}
$$

where $\varphi(r)$ is defined as in Lemma 2.6.

Therefore, Theorem 3.4 follows easily from Lemma 2.6 and (3.6).

As an application, Corollary 3.5 follows immediately from Theorems 3.1, 3.2, 3.3. We remark that the optimal bounds for the complete elliptic integral of second kind obtained from Theorem 3.4 is the same as those from Theorem 3.1 since $\lambda_{1}+\lambda_{4}=1$.

Corollary 3.5. Let $\lambda_{1}=2\left[1-\frac{3}{\pi} \mathcal{E}(1 / 3)+\frac{4}{3 \pi} \mathcal{K}(1 / 3)\right], \lambda_{2}=\log [3 \pi /(9 \mathcal{E}(1 / 3)-$ $4 \mathcal{K}(1 / 3))] / \log 2, \lambda_{3}=[3 \pi-9 \mathcal{E}(1 / 3)+4 \mathcal{K}(1 / 3)] /[9 \mathcal{E}(1 / 3)-4 \mathcal{K}(1 / 3)]$ and $\lambda_{4}=$ $[18 \mathcal{E}(1 / 3)-8 \mathcal{K}(1 / 3)] / 3 \pi-1$. Then the double inequalities

$$
\begin{aligned}
\frac{\pi}{4}\left(1+\sqrt{1-t^{2}}\right) & <\mathcal{E}(t)<\frac{\pi}{2}\left(1-\lambda_{1}+\lambda_{1} \sqrt{1-t^{2}}\right), \\
\frac{\pi}{2}\left(1-t^{2}\right)^{1 / 4} & <\mathcal{E}(t)<\frac{\pi}{2}\left(1-t^{2}\right)^{\lambda_{2} / 2} \\
\frac{\pi \sqrt{1-t^{2}}}{1+\sqrt{1-t^{2}}} & <\mathcal{E}(t)<\frac{\pi \sqrt{1-t^{2}}}{2\left(\lambda_{3}+\left(1-\lambda_{3}\right) \sqrt{1-t^{2}}\right)}
\end{aligned}
$$

hold for all $t \in(0, \sqrt{3} / 2)$.

\section{Acknowledgments}

This research was supported by the Natural Science Foundation of China under Grants 11301127 and the Natural Science Foundation of Zhejiang Province under Grant LY13A010021.

\section{References}

[1] Gh. Toader, Some mean values related to the arithmetic-geometric mean, J. Math. Anal. Appl., 1998, 218(2), 358-368.

[2] M. Vuorinen, Hypergeometric functions in geometric function theory, in: Special Functions and Differential Equations (Madras, 1977), 119-126, Allied Publ., New Delhi, 1998. 
[3] S.-L. Qiu and J.-M. Shen, On two problems concerning means, J. Hangzhou Inst. Electron. Eng., 1997, 17(3), 1-7 (in Chinese).

[4] R. W. Barnard, K. Pearce and K. C. Richards, An inequality involving the generalized hypergeometric function and the arc length of an ellipse, SIAM J. Math. Anal., 2000, 31(3),693-699.

[5] H. Alzer and S.-L. Qiu, Monotonicity theorems and inequalities for the complete elliptic integrals, J. Comput. Appl. Math., 2004, 172(2), 289312.

[6] E. Neuman, Bounds for symmetric elliptic integrals, J. Approx. Theory, 2003, 122(2), 249-259.

[7] H. Kazi and E. Neuman, Inequalities and bounds for elliptic integrals, J. Approx. Theory, 2007, 146(2), 212-226.

[8] Y.-M. Chu, M.-K. Wang, S.-L. Qiu and Y.-F. Qiu, Sharp generalized Seiffert mean bounds for Toader mean, Abstr. Appl. Anal., 2011, 2011, Article ID 605259, 8 pages.

[9] Y.-M. Chu and M.-K. Wang, Inequalities between arithmetic-geometric, Gini, and Toader means, Abstr. Appl. Anal., 2012, 2012, Article ID 830585, 11 pages.

[10] Y.-M. Chu and M.-K. Wang, Optimal Lehmer mean bounds for the Toader mean, Results Math., 2012, 61(3-4), 223-229.

[11] Y.-M. Chu, M.-K. Wang and S.-L. Qiu, Optimal combination bounds of root-square and arithmetic means for Toader mean, Proc. Indian Acad. Sci. Math. Sci., 2012, 122(1), 41-51.

[12] Y.-Q. Song, W.-D. Jiang, Y.-M. Chu and D.-D. Yan, Optimal bounds for Toader mean in terms of arithmetic and contraharmonic means, J. Math. Inequal., 2013, 7(4), 751-757.

[13] W.-H. Li and M.-M. Zheng, Some inequalities for bounding Toader mean, J. Funct. Spaces Appl., 2013, 2013, Article ID 394194, 5 pages.

[14] Y. Hua and F. Qi, The best bounds for Toader mean in terms of the centroidal and arithmetic means, Filomat, 2014, 28(4), 775-780.

[15] H. Sun and Y.-M. Chu, Bounds for Toader mean by quadratic and harmonic means, Acta Math. Sci., 2015, 35A(1), 36-42 (in Chinese). 
[16] Y.-M. Chu, M.-K. Wang and X.-Y. Ma, Sharp bounds for Toader mean in terms of contraharmonic mean with applications, J. Math. Inequal., 2012, $7(2), 161-166$.

[17] Y.-Hua and F. Qi, A double inequality for bounding Toader mean by the centroidal mean, Proc. Indian Acad. Sci. Math. Sci., 2014, 124(4), 527-531.

[18] G. D. Anderson, M. K. Vamanamurthy and M. Vuorinen, Conformal Invariants, Inequalities, and Quasiconformal Maps, John Wiley \& Song, New York, 1997. 